Ann. Biol, anim. Bioch. Biophys., 1979, 19 (3 B), 801-805.

\title{
Influence des facteurs sexuels, chez le rat, sur l'activité des phosphatases alcalines intestinales. Etude immunologique
}

par Nicole FONTAINE

EPHE, Physiologie, Métabolisme minéral des Mammifères, ERA CNRS 070572, Faculté de Pharmacie 92290 Châtenay-Malabry.

Summary. The effect of sexual factors on intestinal alkaline phosphatase activity in rats.

The immunoelectrophoretic method of Laurell has been used for a study of rat intestinal alkaline phosphatase in various sexual conditions. The results showed that ileal alkaline phosphatase depended on the sex and the estrous cycle. Duodenal and jejunal enzymes did not depend on these factors. Both ileal enzymatic activity and calcium retention had the same distribution. Estrous females showed higher values and males lower ones. Diestrous and gonadectomized animals presented values between those of the estrous females and the males.

\section{Introduction.}

L'intensification des échanges calciques s'accompagne dans des circonstances variées d'une activité accrue de la phosphatase alcaline. Pendant le remaniement du test des mollusques où les incorporations du Ca sont augmentées, une augmentation concomitante d'activité phosphatasique est enregistrée (Manigault, 1939). Au cours de la réparation d'une fracture une forte activité phosphatasique apparaît au niveau du cal (Dulce, 1960). Le rat en rachitisme chronique par défaut de facteurs d'utilisation du Ca présente une intensification des échanges calciques de l'os (Dupuis, 1971) à laquelle correspond une exaltation d'activité phosphatasique osseuse (Fontaine ef al., 1977). Dans la muqueuse iléale de rats dont l'absorption est spontanément élevée, s'observe également une activité phosphatasique élevée (Fontaine ef al., 1977).

A maintes reprises nous avons observé que l'absorption et la rétention du calcium, chez le rat, sont fonction du sexe ef du cycle œstral (Fontaine, 1969, 1971, 1973 ; Dupuis et Fontaine, 1978). La femelle de plus de six mois est plus réactive que le mâle et c'est en œstrus que la femelle absorbe ef retient le plus de calcium. Ces différences dans l'aptitude du mâle et de la femelle à utiliser le calcium ne correspondraient-elles pas à des différences d'activité phosphatasique au niveau intestinal? 


\section{Conditions expérimentales.}

Les activités phosphatasiques en divers lieux de l'intestin de rats Wistar, adultes de sept mois, sont étudiées en fonction de l'état sexuel des animaux. Ceux-ci sont choisis de sorte que leur équilibre hormonal soit normal (mâles entiers ou femelles cyclées) ou qu'ils soient soustraits aux influences hormonales sexuelles (animaux gonadectomisés). Les femelles ovariectomisées reçoivent ou non des hormones sexuelles (œstrogènes ef progestérone) par voie sous cutanée.

Les animaux sont sacrifiés au chloroforme. L'intestin est coupé en quatre portions : le duodénum et les trois autres portions jéjunum I, jéjunum Il et iléum étant obtenues en coupant le reste de l'intestin en trois parties égales.

La muqueuse est prélevée par raclage à la spatule sur une plaque réfrigérée. La phosphatase alcaline est extraite de l'homogénat de muqueuse en milieu tamponné (tris- $\mathrm{HCl} 0,02 \mathrm{M}, \mathrm{pH}=7,4 ; 1 \mathrm{mM} \mathrm{MgCl}_{2}$ ) par action du butanol et de l'acétone (Lafont, 1975). Le p-nitrophénylphosphate $(15 \mathrm{mM}$ en milieu tamponné carbonate $30 \mathrm{mM}, \mathrm{pH}=9,8$ ) est le substrat utilisé pour la mesure de l'activité phosphatasique de cette préparation.

Le dosage électroimmunologique employé (Laurell, 1972) est une méthode quantitative qui associe la spécificité de l'immunochimie à la rapidité de l'électrophorèse. II est réalisé sur des dilutions croissantes de solutions de phosphatases semi-purifiées, au sein d'un gel d'agarose (indubiose A 37, IBF) contenant l'anticorps. Celui-ci est produit par un lapin ayant reçu une préparation purifiée de phosphatase alcaline de muqueuse d'intestin grêle de rats impubères. L'électrophorèse détermine des pics dont la hauteur est proportionnelle à la quantité d'antigènes déposés. L'activité enzymatique des immunoprécipités est révélée par une réaction colorée spécifique de la phosphatase alcaline ( $\beta$-naphtyl-phosphate $5 \mathrm{mM}: \mathrm{MgCl}_{2} 1 \mathrm{mM}$; tampon carbonate $30 \mathrm{mM}, \mathrm{pH}=9.8$; Fasi Blue RR Sigma). Les unités d'enzyme par $\mathrm{ml}$ rapportées à la hauteur des pics en $\mathrm{mm}$ s'alignent sur une droite dont la pente est proportionnelle à l'activité moléculaire de l'enzyme.

\section{Résultats.}

La comparaison des mesures directes des phosphatases de l'homogénat de muqueuse de divers lots de rats n'est pas satisfaisante. Les résultats exprimés par rapport aux protéines de la muqueuse sont très variables à l'intérieur d'un même groupe. II n'esł donc pas possible de conclure à une différence entre les lots étudiés par cette méthode.

Au contraire, l'électroimmunodiffusion simple fait apparaître des différences d'activité enzymatique selon le sexe du rat.

Pour l'iléum les expériences sont représentées sur les figures $1 a$ et $1 b$. Elles sont à considérer séparément, le voltage de l'électrophorèse n'étant pas de même valeur (50 $\mathrm{V}$ par plaque pour les animaux groupés par 5 et $25 \mathrm{~V}$ par plaque pour les individuels). Chaque expérience considère des femelles en œstrus et en dicstrus des femelles ovariectomisées et des mâles normaux. De plus, seul un des deux groupes d'expériences porte sur des mâles orchidectomisés ef sur des femelles qui, ovariectomisées, 
reçoivent des hormones (œstradiol et progestérone). Les droites de la figure $1 b$ sont tracées selon les pentes moyennes avec l'écart-type de la moyenne. L'éventail des répartitions des droites traduit une activité enzymatique en rapport avec l'état sexuel des rats. En effet, la pente la plus forte qui correspond à l'activité enzymatique la plus élevée, est donnée par les femelles en œstrus et la pente la plus faible est donnée par les mâles. Entre ces deux valeurs se répartissent graduellement du plus haut vers le plus bas les femelles ovariectomisées recevant des hormones, les femelles en diœstrus, les femelles ovariectomisées et les mâles orchidectomisés.
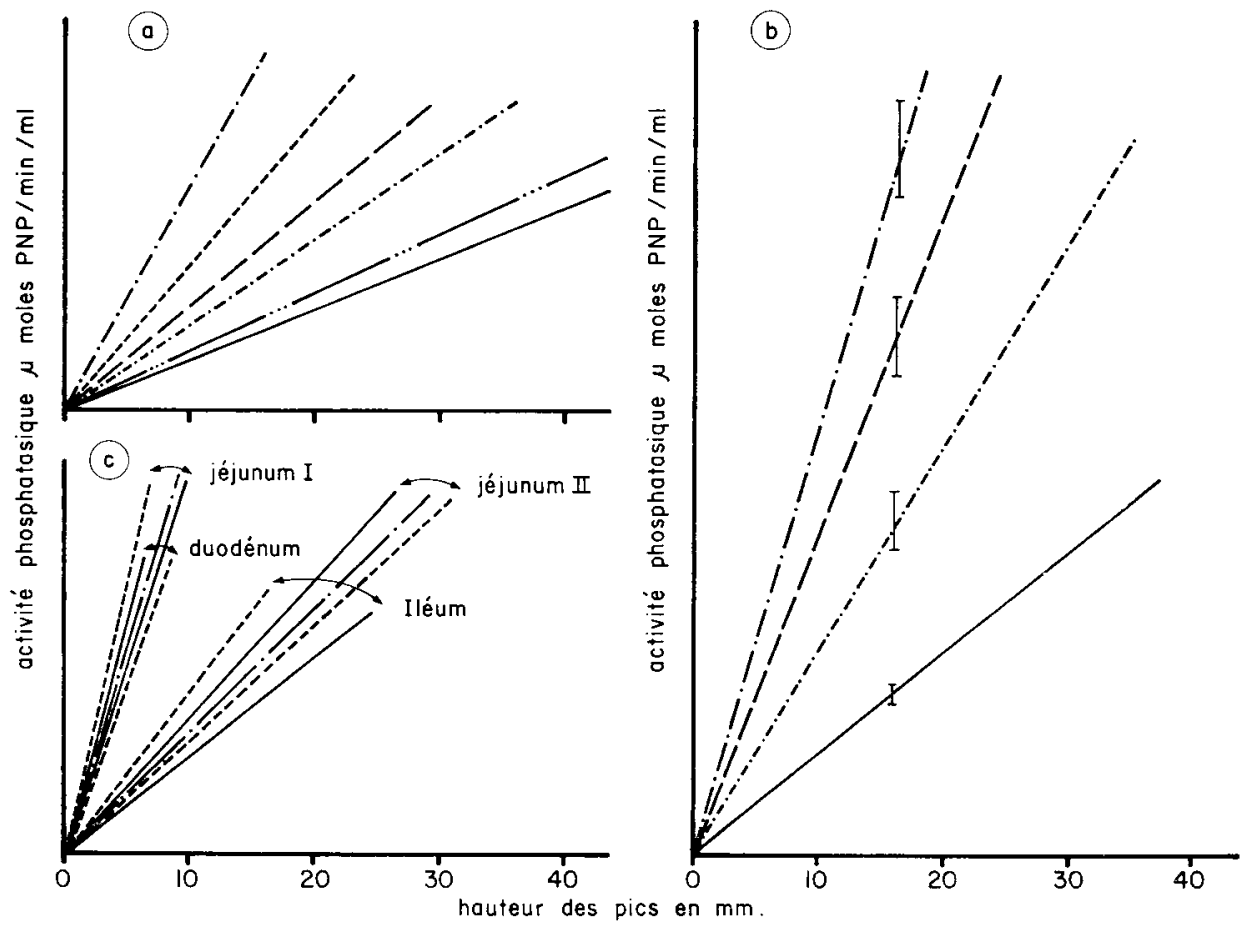

FIG. 1 a-c. - Interprétation graphique de l'électroimmunodiffusion simple de phosphatases alcalines de muqueuses intestinales de rats adultes.

mâles ; - -.. mâles orchidectomisés ; - . . - . - femelles ovariectomisées ; - - - femelles en dioestrus ; -..... femelles ovariectomisées + hormones (œstrogène et progestérone) ; - - - - femelles en œstrus.

FIG. $1 a$ : iléums traités par groupe de 5.

FIG. $1 b$ : iléums traités individuellement $(p<0,01$ entre mâles et femelles ovariectomisées ; $p<0,001$ entre femelles en cestrus et femelles ovariectomisées).

FIG. 1c : muqueuses de diverses parties de l'intestin groupées par 5.

Le schéma n'est pas du tout le même avec le duodénum ou le jéjunum (voir fig. $1 \mathrm{c}$ ). Les pentes des droites obtenues pour chaque lot ne sont pas significativement différentes à l'intérieur de chaque groupe. 


\section{Discussion.}

Cette répartition des valeurs enzymatiques au niveau de l'iléum est à rapprocher de celle de la rétention des alcalino-terreux chez ces mêmes animaux (Fontaine, 1969, 1971, 1973 ; Dupuis et Fontaine, 1978). En effet, pour les rats de onze mois la rétention d'un alcalino-terreux radioactif, le ${ }^{85} \mathrm{Sr}$, mesurée d'après la radioactivité d'un os long (łabl. 1) ou l'absorption de ${ }^{45} \mathrm{Ca}$, déterminée au moyen d'anses iléales injectées in sifu (tabl. 1) ne sont pas de même valeur selon l'état sexuel du rat ef l'éventail de répartition

TABLEAU I

Critères d'ufilisation de l'alcalino-terreux

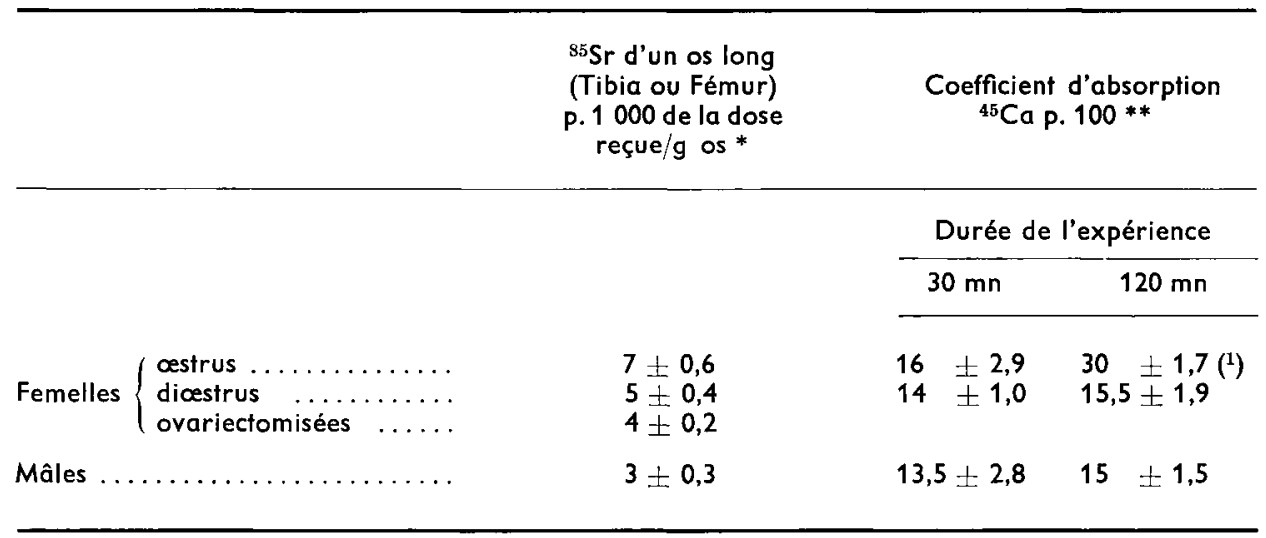

* Le radioélément est administré par sonde cesophagienne.

** Une anse iléale de $12 \mathrm{~cm}$ ligaturée in situ esł injectée de $1 \mathrm{ml}$ d'une solution de $\mathrm{CaCl}_{2} 10 \mathrm{mM}+0,1 \mu \mathrm{Ci}^{45} \mathrm{Ca}$. Après minéralisation de l'anse, on obtient le coefficient d'absorption :

$$
\frac{{ }^{45} \mathrm{Ca} \text { injecté }-{ }^{45} \mathrm{Ca} \text { résiduel }}{{ }^{45} \mathrm{Ca} \text { injecté }} \times 100 .
$$

(1) $F<0,001$.

est superposable à celui obtenu par la mesure au moyen de la réaction immunologique de l'activité de la phosphatase alcaline iléale. Ainsi la ratte adulte utilise mieux le calcium que le mâle, l'absorption, chez cette espèce, se faisant surtout au niveau de l'iléum (Cramer and Coop, 1959). Ces faits s'accordent avec nos présentes observations selon lesquelles certaines qualités de la phosphatase alcaline de l'iléum de la femelle sont supérieures à celle du mâle.

Commission CNERNA Digestion-Absorption/Association des Physiologistes, Paris 5-6 octobre 1978. 


\section{Références}

CRAMER C. F., COOP D. M., 1959. Progress and rate of absorption of radiostrontium through the intestinal tracts of rats. Proc. Soc. exp. Biol. Med., 102, 514-517.

DULCE H. J., 1960. Zur Biochemie der Verknöcherung III. Mineralgchalt Grundsubstanzzusammensetzung und enzymaktivitäten im Callusgewebe und in rachitischen Knochen von Ratten. Hoppe-Seyler's Z. Physiol. Chem., 320,1-20.

DUPUIS Y., 1971. Cinétique de l'utilisation des alcalino-terreux chez le rat âgé constamment carencé en vitamine D. C. R. Acad. Sci., Paris, série D, 272, 1662-1664.

DUPUIS Y., FONTAINE N., 1978. Influence de divers facteurs sur la durée de l'absorption intestinale du calcium chez le rat. Communic. Réun. Assoc. Physiol. J. Physiol., Poris, 74, 27 A.

FONTAINE N., 1969. Etude, au moyen de ${ }^{85} \mathrm{Sr}$, de l'influence du cycle œstral de la ratte sur l'utilisation des alcalinoterreux. C. R. Acad. Sci., Paris, série D, 269, 215-218.

FONTAINE N., 1971 . Incidences sexuelles sur la contamination du rat par $1{ }^{85} \mathrm{Sr}$. Communic 5 e Congr.. int. Radioprotection, Grenoble, 68-77.

FONTAINE N., 1973. Activité du L-arabinose sur l'utilisation du calcium chez la ratte ovariectomisée. C. R. Acad. Sci., Paris, série D, 277, 725-727.

FONTAINE N., FOURNIER P., FOURNIER A., 1977. Nouvelles corrélations entre le métabolisme du calcium et l'activité des phosphatases alcalines intestinales. $27^{\mathrm{e}}$ int. Congr. Physiol. Sci., XIII, $n^{\circ}$ 676, 232.

LAFONT J., 1975. Rôle métabolique des phosphatoses alcalines ou niveau de l'entérocyte. Thèse Doct. Ełat. Univ. Sci. Techn. Languedoc, Montpellier.

LAURELL C. B., 1972. Electroimmuno assay. Scand. J. clin. Lab. Invest., 29, Suppl. 124, 21-37.

MANIGAULT P., 1939. Recherches sur le calcaire chez les Mollusques. Phosphatase et précipitation calcique, histochimie du calcium. Ann. Inst. Océan., 18, 331-426. 